\title{
Effective quadrature formula in solving linear integro-differential equations of order two
}

\begin{abstract}
In this note, we solve general form of Fredholm-Volterra integro-differential equations (IDEs) of order 2 with boundary condition approximately and show that proposed method is effective and reliable. Initially, IDEs is reduced into integral equation of the third kind by using standard integration techniques and identity between multiple and single integrals then truncated Legendre series are used to estimate the unknown function. For the kernel integrals, we have applied Gauss-Legendre quadrature formula and collocation points are chosen as the roots of the Legendre polynomials. Finally, reduce the integral equations of the third kind into the system of algebraic equations and Gaussian elimination method is applied to get approximate solutions. Numerical examples and comparisons with other methods reveal that the proposed method is very effective and dominated others in many cases. General theory of existence of the solution is also discussed.
\end{abstract}

Keyword: Approximations; Collocation method; Integro-differential equations; Legendre polynomials 